\title{
Aproximación a los cambios percibidos en los servicios sociales por sus gestores: una adaptación impuesta
}

\section{Marta García-Domingo}

Área de Trabajo Social y Servicios Sociales, Universidad de Jaén

mgdoming@ujaen.es

Urruti geratu da, jadanik, ongizate sozialaren berezko osagai zuten ongizate-estatuen "urrezko aroa”. Panorama horren aurrean, artikulu honetan proposatzen da aktoreek hautemandako aldaketen berri izatera (arazo sozialen definizio eta konponketan inplikatutako aktore sozialak), eta modu horretan ahuldade eta mehatxuak identifikatzea. Gure inguruko herrialde batzuetako informatzaile garrantzitsuekin buruturiko sakoneko elkarrizketen azterlana oinarri hartuz, egiaztatzen da ongizate sozialerako garrantzi handiko aldaketen presentzia eta Europar Batasunaren hedakuntzaren esparruan gertatzen ari diren joera berriak, eta premiakoa gertatzen da bi prozesu horiek nabarmendu eta gogoeta burutzea.

\section{GAKO-HITZAK:}

Europar Batasuna, gizarte-ongizatea, krisialdi sozioekonomikoa, aldaketak, mehatxuak.
Lejos queda ya la "edad dorada" del bienestar social, en la que los Estados de Bienestar se conformaban como un componente intrínseco del capitalismo de la posguerra. Ante el nuevo panorama, este artículo propone una aproximación a los cambios percibidos en el bienestar social por sus propios gestores (actores sociales implicados en la definición y resolución de los problemas sociales), que permita identificar sus debilidades y amenazas. A partir del análisis de entrevistas en profundidad a informantes clave en diversos países de nuestro entorno, se constata la presencia de cambios transcendentales para el bienestar social así como de nuevas tendencias en expansión en el marco de la Unión Europea que necesitan ser evidenciados y reflexionados.

\section{Palabras Clave}

Unión Europea, bienestar social, crisis socioeconómica, cambios, amenazas. 


\section{Introducción}

El contexto actual se halla marcado por las profundas transformaciones en el ámbito de la política social y los servicios sociales (Fundación Foessa, 2014). Destaca, asimismo, la presencia de un proceso de convergencia supranacional que provoca una reestructuración en los Estados y sus políticas tanto macroeconómicas como sociales (Moreno, 2001). En este proceso de convergencia, se observa una reducción del sector público en los diferentes estados miembro, que fue predicho por Mishra (1996) hace ya casi dos décadas. Este declive es consecuencia de una serie de factores políticos e ideológicos que han priorizado cuestiones vinculadas al mercado, el individualismo y la competitividad, con el consiguiente distanciamiento evidente de los valores de igualdad y universalidad. La crisis económica internacional iniciada en 2008 ha tenido una influencia considerable en este proceso. En algunos casos, los recortes sociales y el consecuente debilitamiento de los Estados de Bienestar han sido el resultado de un contexto social adverso. En otros, ha sido fruto del imperativo de austeridad marcado por la propia Unión Europea y sus países "vencedores" o menos afectados. Por último, destaca la enorme influencia del neoliberalismo y la penetración de nuevas concepciones de bienestar social, que cambian los modelos e incluso cuestionan las teorías que los sustentan.

Centrando la atención en el último factor motivador de las transformaciones sociales mencionado, cabe destacar que en las últimas décadas se ha producido un apogeo del discurso neoliberal que rompe con la hegemonía keynesiana. Este discurso se fundamenta en la supuesta existencia de una intervención excesiva del Estado, considerando pertinente reforzar el rol del mercado. Dicho de otro modo, el pensamiento neoliberal asume que la globalización económica y la internacionalización de los mercados financieros conducen a los gobiernos de la UE y de la OCDE a desarrollar unas políticas públicas reductoras del Estado del Bienestar, con la consiguiente minoración en la protección social e incremento de la flexibilidad laboral. Aunque -especialmente en sus inicioslas ideas neoliberales tienen un especial calado en Reino Unido y encuentran resistencia en la mayoría de países europeos (Mishra, 1992; Navarro, 1997), en los últimos años su resurgimiento y expansión es prácticamente generalizado, resultado de la política de contención del gasto público adoptada por muchos países de la Unión Europea. En este sentido, aunque la tesis del desmantelamiento del Estado de Bienestar viene apuntándose desde finales de los setenta, aludiéndose a las profundas modificaciones ideológicas e institucionales acaecidas (RodríguezCabrero, 1989), en el contexto actual identificamos una reestructuración de dicho desmantelamiento. Ello supone un reto para las ciencias sociales, ya que provoca dualización social y rigidez en la estructura social, así como una preocupante indefensión social. En este sentido, como recogen García-Domingo y Sotomayor (2016), el debilitamiento de los Estados de Bienestar y el incremento de las desigualdades y la exclusión social son fenómenos interrelacionados con una relación causa-efecto bidireccional: el debilitamiento de los Estados de Bienestar (causa) produce y reproduce situaciones de exclusión y, por tanto, acentúa la polarización (efecto). Por otra parte, el aumento de las situaciones de desigualdad y exclusión social (causa), provoca un colapso en los sistemas de bienestar que reduce su capacidad garantista de protección y seguridad (efecto).

En concreto, en este artículo analizamos el sistema público de servicios sociales, considerado el cuarto pilar del bienestar social desde su impulso con el Sistema para la Autonomía y Atención a la Dependencia (junto a la educación, la sanidad y la seguridad social). Centrando la atención en el caso español, los principios inspiradores del sistema de servicios sociales, que - al menos teóricamenteorientan sus servicios y prestaciones, quedando recogidos en las leyes autonómicas de servicios sociales, son: universalidad, responsabilidad pública, igualdad, equidad, prevención y dimensión comunitaria, promoción de la autonomía personal, atención personalizada e integral, inserción y normalización, proximidad, solidaridad, calidad, participación, cooperación y colaboración. No obstante, el sistema público de servicios sociales español se ha caracterizado por su debilidad y desarrollo limitado en comparación con los otros sistemas - pensiones, salud y educación-. Incluso en épocas de prosperidad, el desarrollo económico no ha ido acompañado de un desarrollo social acorde al contexto de crecimiento. El Plan Concertado supuso un considerable avance en materia de servicios sociales municipales, contribuyendo a la ampliación y la consolidación de prestaciones -económicas y de servicios- y a su universalización (Las Heras, 2002; Subirats, 2004). Partiendo de la descentralización autonómica de las funciones de servicios sociales, la reciente aprobación de la Ley 9/2016, de 27 de diciembre, de Servicios Sociales de Andalucía, supone un importante hito en la materia. Sin embargo, la propia ley favorece la privatización en el ámbito de servicios sociales -estableciendo unos estándares de calidad-, institucionalizando la actual tendencia a ella.

Junto a esta tendencia a la privatización de la cuestión social, también se identifica una mayor presión social. En definitiva, como consecuencia de la globalización neoliberal y sus imperativos de eficiencia económica, las políticas sociales están marcadas por la presión, la coerción y la exigencia de responsabilidades, entre otros factores, lo que redunda en una merma en los derechos de la ciudadanía. Estos cambios sociales se traducen en una serie de cambios en la gestión de servicios sociales que se han visto acentuados notablemente en la última década. Algunos de estos cambios son percibidos y apuntados por los/as propios/as usuarios/as de estos servicios, por los/as analistas sociales e incluso por los/as técnicos/as que desarrollan su labor profesional en ellos, como 
quedará evidenciado a lo largo de este artículo. Es por ello por lo que apostamos por reflexionar con los entrevistados acerca de los cambios detectados desde su posición privilegiada para analizar la evolución de estos servicios.

\section{Método}

Como queda reflejado en la introducción, son muchos los autores que apuntan cambios notorios en materia de bienestar social y un debilitamiento del sistema público de servicios sociales. Para el análisis de esta realidad hemos empleado una metodología cualitativa, a partir de la técnica de la entrevista en profundidad semiestructurada y la observación participante. Ambas técnicas se han desarrollado en tres contextos que representan los principales modelos de bienestar social presentes en la Unión Europea. En concreto, seleccionamos el modelo centroeuropeo (representado por Alemania), el nórdico (representado por Suecia y Finlandia) y el mediterráneo (representado por España). Llevamos a cabo un total de 48 entrevistas $^{1}$ (más seis de validación) a actores sociales implicados en la definición y respuesta técnica e institucional a las necesidades sociales. En concreto, entrevistamos a expertos/as de tres perfiles profesionales considerados relevantes para el análisis propuesto: académicos/as e investigadores/as de reconocido prestigio por su abordaje de la materia; trabajadores/as sociales; y representantes políticos e institucionales. En base a ello, se ha llevado a cabo un análisis comparativo de discurso entre contextos y grupos de expertos/as, que permite extraer tendencias comunes y diferenciales entre contextos al respecto de los cambios acaecidos. Cabe mencionar la larga trayectoria profesional de la mayoría de los participantes, que les permitió adentrarse en dicha reflexión desde una perspectiva longitudinal.

\section{Análisis}

En este apartado reflejaremos los principales resultados hallados en la presente investigación con respecto a los cambios en materia de bienestar social. En concreto, reflexionaremos sobre dos categorías básicas que integran la mayor parte de los hallazgos resultado del análisis de las entrevistas en la materia: la categoría restricciones (apartado 3.1. Restricciones en los sistemas de bienestar social europeo); y la categoría privatización/concertación (apartado 3.2. La concertación de los servicios sociales públicos).

${ }^{1}$ Las entrevistas se codifican con dos letras (una mayúscula y una minúscula) y un número, cada uno de ellos separados por punto. La letra mayúscula corresponde al país en que se contextualiza la entrevista $(A=$ Alemania; $S=$ Suecia; $F=$ Finlandia; y $E=$ España). La segunda letra se corresponde con el perfil de informante clave $(a=$ académico; $r=$ representante institucional; y $p=$ profesional de atención social). Por último, un número del 1 al 48, correspondiendo la numeración 1 a 19 a la zona 1 (Alemania), 20 a 37 a la zona 2 (Suecia y Finlandia) y 38 a 48 a la zona 3 (España).
Es necesario mencionar que el concepto de servicios sociales es diferente en función de si nos centramos en España o en la Unión Europea. En relación con ello se detecta un empleo diferencial del término "servicios sociales": mientras que en España sigue prevaleciendo una acepción restringida de los servicios sociales (vinculada al llamado "cuarto pilar” del Estado de Bienestar), en Alemania y Suecia predomina una acepción amplia. Esto hace que en determinados momentos de las entrevistas estas hagan alusión a realidades distintas, lo que ha sido tenido en consideración durante el análisis.

“El termino servicios sociales también es confuso en nuestro país, nosotros entendemos por servicios sociales los servicios sociales comunitarios, mientras que en el ámbito anglosajón los servicios sociales son todos los servicios de responsabilidad pública que se dirigen a la población, ¿no? Entonces depende del concepto que estemos manejando, yo creo que los servicios sociales en términos generales se dirigen a toda la población es lo que pasa, que los servicios sociales comunitarios en España han estado focalizados y dirigidos a una población en situación de exclusión social.” (E.a.44)

Tras esta puntualización conceptual, cabe afirmar que presenciamos un notorio debilitamiento de los sistemas públicos, en general, y del sistema de servicios sociales (concepción restringida), en particular.

“Creo que en la actualidad no ayudamos a la gente tanto como antes. Lo dejamos estar, y las diferencias de clase son mucho mayores que hace quince años." (S.a.25)

Aunque con consecuencias, presencia y connotaciones diferenciales entre países, identificamos aspectos comunes que están modificando la estructura, gestión y funcionamiento de los sistemas de servicios sociales europeos. A su vez, detectamos aspectos disímiles entre zonas en lo relativo a estos cambios. A continuación se exponen los más significativos.

\subsection{Restricciones en los sistemas de bienestar social europeos}

A nivel general, se evidencia un cambio importante en el sistema de servicios sociales, con importantes consecuencias en la intervención social. Según nuestros expertos/as, el sistema se ha impregnado de un carácter limitado, restrictivo, controlador y burocrático. Con respecto a las causas de estas restricciones, existen interpretaciones diferentes en función de la zona ${ }^{2}$ analizada. En las zonas 1 y 2 los informantes perciben que los cambios producidos no

\footnotetext{
2 Zona 1: Centroeuropa (representado por Alemania) Zona 2: países nórdicos (representado por Suecia y Finlandia) Zona 3: Banda mediterránea (representada por España)
} 
son tanto resultado de la crisis, sino más bien de un cambio ideológico. Por su parte, los actores sociales de la zona 3 vislumbran una clara relación entre el cambio de gestión y naturaleza de los servicios sociales y la crisis socioeconómica internacional.

En esta línea, se ponen de manifiesto realidades diferentes según la zona analizada, aunque con un claro punto convergente: el endurecimiento del sistema y la reducción de garantías.

En el caso de Suecia, el endurecimiento del sistema de seguridad social y la reducción de garantías afectan especialmente a sus tradicionales beneficiarios directos; es decir, a los que durante años, o incluso décadas, han recibido prestaciones y servicios bajo prescripción profesional y con un seguimiento y “control” más permisivo o flexible. En este contexto, resulta pertinente hacer referencia a las pensiones enfermedad o invalidez, que han experimentado un endurecimiento considerable:

“Ahora para obtener bienestar social hay muchas más restricciones cuando estás enfermo. Te dicen 'vale, no puedes andar, pero puedes hacer equis'. Esto hace que todo sea mucho más difícil y más duro. Mucha gente que recibía estas pensiones ya no las tienen ni las van a tener más. Se les dice 'ya la has tenido suficiente', pero esa persona no está bien y entonces no tiene nada." (S.r.20)

Las consecuencias de esta rigidez son sufridas a diario por un creciente número de familias y personas con problemas de salud en Suecia. Lo que a nivel estadístico se pone de manifiesto a través de un incremento de la desigualdad y la pobreza, a nivel personal se evidencia en las duras condiciones de vida experimentadas por aquellos que durante años -0 incluso décadas- han permanecido en este sistema sin vinculación al mercado laboral. En muchos casos, sus herramientas de inserción laboral se han visto mermadas y las posibilidades de "hacerse hueco" en el mercado laboral altamente competitivo son escasas. A ello se suman unas condiciones de salud que, a menudo, les impiden el desarrollo normalizado -0 exigido- de las funciones demandadas. Los costes de salud, sociales y personales son evidentes. En este contexto, cabe deducir que la salud -o más bien su ausencia- supone cada vez más un riesgo de que se den situaciones de carencia o privación. Aunque la inserción social sería una consecuencia esperable de la política de activación, en la realidad nos encontramos con que provoca flujos exclusógenos, al no existir una verdadera adaptación de la red de inclusión laboral.

Aunque existen otras vías para dar respuesta a aquellos que se hallan en un limbo institucional tras este cambio - fuera del sistema de seguridad social y también del laboral-, las opciones son limitadas, coercitivas y estigmatizadoras. Más vinculadas a los servicios sociales en su sentido estricto, tienen una serie de deficiencias e inconvenientes que son reflejadas en los discursos.
“Antes si estabas enfermo y un médico lo certificaba, tú obtenías una pensión del sistema. Toda la gente que recibía esta pensión fue citada para ir a hacerse pruebas y mucha gente perdió su pensión. Pero no habían trabajado durante muchos años, así que necesitan financiación de los servicios sociales. [...] pero dan mucho menos. Tienes que solicitarlo cada mes, así que no saben si lo van a conservar el siguiente mes. Tienen que cumplir unos requisitos. Por ejemplo, si no tienen trabajo tienen que solicitar los trabajos que salen y si no lo hacen, no reciben el dinero. Además, están recibiendo servicios sociales y eso provoca vergüenza. Por tanto, ha habido un cambio de status y exclusión social.” (S.r.22)

Cabe destacar, además, que el proceso informativo estuvo marcado por la confusión, el desconcierto y la incomprensión por parte de los destinatarios. Asimismo, los discursos reflejan una tendencia a la deshumanización y reducción de la empatía por parte de los gestores del sistema, que a su vez se hallan condicionados por las directrices impuestas. Con respecto al sistema de servicios sociales (acepción restringida), también se aprecia el endurecimiento de los requerimientos exigidos para ser beneficiario de las prestaciones disponibles, así como la de burocracia asociada y el control del cumplimientos de los requisitos.
"La cantidad de dinero que ganas es muy limitada, así que el sistema no está diseñado para pertenecer en él mucho tiempo. Ellos no pueden tener nada en propiedad. [...] Hay demasiado control y demasiada documentación. Esto también es nuevo. Tienes que documentarlo todo mucho más y la aplicación utilizada es cada vez más compleja de entender y necesitas que los clientes todo el rato cumplimenten documentos [...]." (S.r.22)
"Hoy en día no obtienes nada. Sólo control y sanciones. No te damos dinero porque no haces esto, o porque no cumples este requisito, o porque no has venido a la cita... Es diferente.” (S.p.29)

Se impone, por tanto, un sistema marcado por las restricciones, el control, la burocracia y la aplicación de sanciones. En definitiva, la manera de enfrentar los problemas sociales se torna mucho más estricta, reflejándose en cuestiones como: la limitación temporal de la intervención; la concepción limitada de los problemas, las necesidades e incluso recursos (lo que no está definido, no existe); la falta de acceso motivada por el endurecimiento de los requisitos marcados para su obtención, que deja a un creciente número de personas en un "limbo social"; y el control para garantizar el cumplimiento de esos límites, entre otras.

"Tienes que probar tus necesidades, y si esas necesidades no existen en el mapa de necesidades, es tu problema. El sistema no es lo que era.” (S.a.25) 
En este sentido, no parece arriesgado afirmar que las transformaciones en el sistema de bienestar social modifican su propio carácter, alejándolo de sus principios inspiradores, así como su funcionamiento y capacidad de reacción/intervención. Por otro lado, cabe destacar que estas limitaciones no solo afectan a los potenciales beneficiarios del sistema, sino también a los que trabajan en él. El avance de las políticas de austeridad, tanto en extensión como en influencia, marca el funcionamiento de los servicios públicos y la actuación de los profesionales. En definitiva, asistimos al retroceso de uno de los modelos que durante décadas ha sido referente del progreso social: el modelo sueco. Una retracción que, pese a no haber sido suficientemente reflexionada a nivel internacional, está integrada en el discurso de los expertos suecos, así como por la sociedad escandinava en su conjunto. En este sentido, el análisis de un discurso evidencia un posicionamiento mayoritario contrario a los cambios detectados, según el cual las restricciones suponen un riesgo y una amenaza para la sociedad universalista. No obstante, también hay un grupo, minoritario pero en expansión, que concibe el cambio como necesario, bajo la consideración de que el anterior sistema generaba dependencia de lo público.

"Yo creo que quizás tengamos que cambiar. Probablemente necesitamos cambiar. La sociedad ha cuidado demasiado de sus ciudadanos hasta ahora. Así que probablemente sea positivo reducir esto, pero yo creo que los cambios están siendo demasiado rápidos. Los ciudadanos suecos dependen mucho de la sociedad. La gente viene y dice 'me voy a separar de mi marido y quiero un apartamento para vivir' y nosotros decimos 'no' y ellos se enfadan... Porque siguen creyendo que la sociedad se tiene que responsabilizar de esto, porque así ha sido hasta ahora. Y es duro para los trabajadores sociales explicarlo." (S.r.22)

Por otro lado, en Alemania (zona 1) hallamos una influyente tendencia a la activación laboral (tendencia generalizada en la Unión Europea, con mayor peso en la sociedad alemana). En este contexto, los individuos son percibidos como "sujetos" que tienen que resultar productivos para su país. Por encima de las circunstancias personales o de las aspiraciones individuales, se halla la disposición (incentivada o impuesta) a participar en el sistema productivo. Motivado por esta tendencia, la actual intervención con individuos en edad de trabajar en Alemania se centra, casi de manera exclusiva, en el seguimiento y control de la búsqueda activa de empleo, quedando relegadas a un segundo plano otro tipo de intervenciones. Las entidades del tercer sector reclaman una intervención pública más holística, ya que en la práctica son estas entidades no lucrativas las que acompañan al individuo en su proceso de inserción sociolaboral.

El gran cambio señalado en Alemania se refiere a la implantación del sistema Hartz (en la actualidad, Hartz IV), que engloba tanto los principios inspiradores y su fundamento, como la organización, gestión, rol profesional y requisitos de acceso.

Además, se modifica el rol de los profesionales intervinientes y se diversifica el de los usuarios o demandantes implicados en el proceso.

En España (zona 3), la principal deficiencia señalada es la insuficiencia de recursos, que ha paralizado el progreso en materia de bienestar social conseguido en la década previa a la crisis y supuesto un retroceso sin precedentes.

"No creo que el sistema de Servicios Sociales en España sea tan malo... lo malo es que no tiene recursos. Lo que no puede ser es que un sistema de servicios sociales, que creo que su estructura es bastante aceptable, pero que estén faltos de recursos para cumplir con sus objetivos.” (E.r.41)

Resulta paradójico que justo cuando y donde los servicios sociales son más necesarios para atender a un número cada mayor de personas con problemas y a la radicalización de dichos problemas, se produzca en el sistema un recorte sin precedentes desde su implantación. Por tanto, en un contexto de creciente desigualdad, pobreza y exclusión social, la demanda de servicios sociales públicos aumenta, pero el sistema no puede dar una respuesta efectiva a las necesidades de la población.

\begin{abstract}
"Se pone en primera línea de batalla, al combate de la crisis, a un sistema que es en sí mismo precario [...]. Y... bueno, los servicios sociales son un sector que teniendo la obligación de atender los efectos de la crisis, está soportando en sí mismo la crisis de una manera brutal. Y esto no deja de ser una paradoja: el sistema de apoyo a los afectados por la crisis también está en crisis." (E.a.44)
\end{abstract}

\subsection{La concertación de servicios sociales públicos}

Por otro lado, resulta necesario reflexionar sobre un fenómeno con un peso creciente en las sociedades occidentales desarrolladas: la privatización o concertación de servicios públicos. La importancia progresiva de este fenómeno queda reflejada en las entrevistas, siendo muchos los/as entrevistados/as que se refieren a él como una consecuencia directa de la crisis en la estructura y funcionamiento de los servicios de bienestar social. En este sentido, mientras algunos sitúan el punto de inflexión de esta privatización en la crisis de los noventa, otros (especialmente en la zona 3) lo sitúan en la actual crisis socioeconómica. No obstante, el debate entre lo público y lo privado en servicios sociales no resulta, ni mucho menos, novedoso. Es, por tanto, un viejo debate con nuevas connotaciones.

En pleno siglo XXI, resulta impactante que, lejos de haber avanzado en el reconocimiento de la importancia de los servicios sociales públicos, la lucha por su defensa esté en pleno apogeo por la 
pérdida de derechos acaecida. Cabe destacar que las nuevas tendencias y corrientes neoliberales que están penetrando en el sistema no son fruto únicamente de la crisis socioeconómica, sino que ya a comienzos de siglo, la Comisión de Servicios Públicos y Comercio Internacional de ATTAC España (ATTAC España, 2004: 7) emitía su Informe sobre la privatización de los servicios públicos con el fin último de "ofrecer argumentos prácticos a la ciudadanía que quiera involucrarse activamente en las luchas para la mejora de los servicios públicos". En esta línea, identifica las siguientes consecuencias de la privatización de servicios garantes de derechos básicos: el aumento de los costes para los usuarios, los obstáculos a la universalidad en el acceso y la degradación de la calidad (ATTAC España, 2004).

En las entrevistas se aprecia claramente una defensa de los servicios públicos, que se extiende incluso entre representantes de instituciones sin ánimo de lucro, que pese a estar prestando servicios de carácter público desde el ámbito privado, realizan un análisis crítico de la coyuntura actual y de su rol en ella.
“Los servicios públicos están bien dotados de personal, bien estructurados en cuando a su campo de actuación y lo que falta son recursos, y los poderes públicos no los proporcionan porque existen entidades que los están proporcionando. Yo creo que el $99 \%$ de los servicios que está prestando Cáritas los debería prestar la Administración Pública, que es su obligación por ley. Pero les resulta más fácil darnos una subvención para que nosotros lo hagamos porque sabe que el costo de ese servicio es el $50 \%$ más barato que el que ellos pudieran prestar.” (E.r.41)

La consideración al respecto es clara y prácticamente unánime, defendiéndose la responsabilidad y gestión pública de estos servicios de bienestar social. No obstante, en caso de concertación, los/as expertos/as coinciden en mostrar una mayor confianza hacia la opción de concierto con entidades sin ánimo de lucro, en el cual los posibles beneficios monetarios se reinvierten (o al menos deberían reinvertirse) en el bienestar de la población.

"Pero entiendo que si la entidad es no lucrativa, la gestión debe ser mejor. Al final una empresa lo que va a buscar es el beneficio. Una ONG, no. Incluso si hubiera beneficio se va a reinvertir para trabajar con la población. Una empresa privada, no. Entonces yo entiendo que debería priorizarse a las organizaciones sin ánimo de lucro.” (E.p.42)

"Yo prefiero instituciones especializadas en trabajo social, instituciones sociales que, aunque por supuesto ganen dinero, no persigan ese fin. Prefiero las instituciones sin ánimo de lucro, aunque también tienen sus problemas, por supuesto. A mí hay cosas que hacen las ONG que no me gustan; por ejemplo hay veces que usan el dinero para ellos mismos y no para los clientes.
Pero, en teoría, no debería ser así, su perfil no es lucrativo." (A.r.10)

Los dos argumentos clave de oposición al concierto de estos servicios son: la pérdida de calidad que llevan aparejados y la irrupción de los principios mercantiles (por ejemplo, ley de la oferta y la demanda, priorización de los precios, etc.) en asuntos de interés general y responsabilidad social.

\begin{abstract}
"No creo que sea una buena alternativa, porque no solo son de bajo coste, sino también de baja calidad. Esto no puede permitirse. No puede permitirse que los principios del mercado entren en esta área de responsabilidad social. Según la ley, las instituciones públicas tienen que buscar el proveedor más barato. Afortunadamente, aún este principio se equilibra con la calidad de los servicios también, es decir, que no todo es el precio. Por tanto, por ahora está equilibrado de alguna manera, aunque cada vez se le da más peso al precio." (A.a.4)
\end{abstract}

“Lo público no necesita obtener beneficios y una empresa privada que intenta abaratar costes pretende obtener un beneficio, así que el ahorro tiene que estar en algún lado.” (E.r.46)

Advierten, además, de que el aumento de servicios privados acentúa la desigualdad de oportunidades y la polarización.

“Nada local, nada municipal, nada estatal, todo privado. Todo se está privatizando, incluso los colegios. Nosotros siempre habíamos estado orgullosos de Suecia, porque nosotros no éramos como Inglaterra, todos los niños iban al colegio público, en el que los niños ricos y los niños pobres compartían aulas. Pero ya no es así. Hay colegios públicos, gratuitos, a los que las familias ricas llevan a sus hijos y se pagan con los impuestos públicos. Por tanto, mis impuestos ayudan a los ricos a tener una educación mejor. Es tan cínico. Los pobres se quedan fuera porque no hay plazas. Los ricos tienen contactos... y así lo consiguen." (S.p.31)

Con respecto a los perjudicados por esta tendencia privatizadora, no solo se apunta a los beneficiarios directos de los servicios objeto de privatización, sino también a sus gestores. Tanto unos como otros presencian una merma en la calidad: los primeros, en los servicios recibidos; los segundos, en sus condiciones laborales. Avalando esta afirmación, una de las entrevistadas visibiliza la realidad de un creciente número de profesionales que trabajan para garantizar el bienestar y seguridad de los ciudadanos a los que atienden, hallándose su propio bienestar y seguridad en riesgo. Las consecuencias de esta privatización no solo afectan a los usuarios de los servicios, que perciben una merma en la calidad, sino también en las personas que los gestionan, cuyas condiciones laborales también sufren un importante deterioro. 
“Lo que realmente ha cambiado es que antes gestionaban lo que creían necesario y la Administración Local o Federal se lo pagaba. Ahora todo sale a concurso a través de concurrencia y diferentes empresas quieren desarrollar los programas. Y esto es un problema para los servicios sociales y lo que hacen es disminuir la seguridad de los trabajos. Hay muchos trabajadores en servicios sociales que tienen contratos reducidos, minijobs. Trabajan por la seguridad de la gente, pero sus propias condiciones de seguridad han cambiado. Por tanto, las cosas no están bien.” (A.a.3)

“La externalización no es sino una forma de privatización de los servicios públicos, que son más eficaces y más baratos, ¿a costa de qué? Porque si son más baratos es porque los profesionales cobran menos o que las calidades de los servicios que se prestan es peor.” (E.a.44)

“La calidad se está viendo afectada porque si ofreces estos servicios con un coste bajo, probablemente no pagues a tus trabajadores bien y por supuesto esto afecta a la calidad del trabajo. Hay mucha gente que hace su trabajo en estas empresas y luego se cambian a otras. En definitiva, hay una afectación de la calidad.” (A.p.14)

Entre las consecuencias más fácilmente perceptibles, nos encontramos con una creciente movilidad profesional, que afecta a la seguridad de dichos profesionales y, a su vez, genera desconfianza en los usuarios. Por ejemplo, una de las entrevistadas en Suecia reflejaba las fluctuaciones de personal de apoyo y asistencia que se producen en el servicio de ayuda a domicilio, como consecuencia de su privatización. Esto genera desconfianza e inseguridad en un colectivo vulnerable que, en un sentido literal, abre las puertas de sus casas a un "apoyo" que perciben como inseguro y desconcertante, alejándose de su propósito.

“Esa persona viene y te ayuda y tú estás un poco asustada porque hay gente nueva viniendo cada día. No sabes quiénes son. Y eso no es una buena situación de cuidado. Y vemos eso en el cuidado de personas mayores. En una semana hay tres personas que vienen a tu casa y esto no te hace sentir segura." (S.p.33)

Los entrevistados de la zona 2 manifiestan que la privatización está afectando especialmente a los servicios sanitarios o de cuidados de salud y atención a la dependencia.

“Los servicios sanitarios son bastante débiles en Finlandia. Los servicios de salud no funcionan tan bien para la gente que no trabaja. Los trabajadores tienen buenos servicios porque trabajan. Se habla de universalidad de los servicios, pero los servicios de salud se están debilitando y el acceso a los servicios no es igualitario.” (F.a.35)
No obstante, no son los únicos que evidencian la privatización de los servicios de cuidado, sino que es una realidad manifestada en las tres zonas objeto de estudio.

En Suecia se refiere la privatización de las medidas destinadas a la inclusión social de refugiados e inmigrantes (por ejemplo, los programas de idiomas). Aunque esto también sucede en Alemania, no tiene la misma presencia en las entrevistas ni se reivindica con el mismo arranque en la zona 1.

“Luego tenemos los programas de enseñar sueco a refugiados e inmigrantes. Desafortunadamente es una de las acciones que están sufriendo la intervención del mercado. Ellos pagan a las compañías para que den estas clases de sueco. Hay competencia entre empresas y las ofrecen más y más baratas, con lo que pierden calidad. Esta forma de pagar a las empresas para que lo hagan hace que la calidad de los servicios sea cada vez menor." (S.a.28)

Como alternativa intermedia, algunos/as expertos/as proponen que la privatización de servicios sociosantitarios se acompañe de exigencias mínimas de calidad. La idoneidad de la prestación de servicios tendrá que ser supervisada de forma rigurosa por la entidad pública responsable de su concertación, a través de técnicas de evaluación acordes. En definitiva, la externalización tendría que estar acompañada de una regularización mucho más exhaustiva, que garantice unos estándares de calidad y que beneficie tanto a los/as usuarios/as de los servicios como a los/as prestadores/as (condiciones de contratación, etc.).
"Podría ser una buena alternativa si se exigen unos mínimos de calidad y si se tiene una tutela desde las Administraciones Públicas segura y fuerte. No significa que esto no lo pueda hacer nadie si no es desde lo público. Hay empresas que trabajan muy bien y que no trabajan a cualquier coste y aun así suelen ser más baratas que las Administraciones Públicas. Pero en cualquier caso tienen que estar tuteladas y bajo la supervisión estricta de la Administración Pública, por el bien del ciudadano, no por otra cosa." (E.r.45)

Como principal aspecto positivo que podría entrañar una privatización rigurosamente vigilada y evaluada, se señala la mayor fluidez:
"Si todos los servicios los tuviera que prestar el Estado sería más lento, ya que no se podría atender a un determinado tipo; por lo tanto, a lo mejor no todo tiene que ser así, lo público se puede hacer de muchas maneras.” (E.a.47)

Con respecto a la reducción del gasto público que supone esa privatización, se muestra una oposición tajante: no pueden reducirse gastos a costa de la calidad de los servicios sociales.

"Tiene que estar integrado en cumplir las normas en obligarlo a cumplir y en pagarlo bien también; 
o sea, lo que no puede ser es que yo sepa que un servicio cuesta cien y cuando lo saco a lo privado lo saco en veinte, porque estoy por la puerta de atrás sabiendo que el que lo saque lo hará por quince porque además le va a sacar cinco." (E.a.47)

Sin bien los/as expertos/as reclaman y apuestan por la implementación de nuevas fórmulas, así como la mejora y perfeccionamiento de otras ya implantadas, en ningún momento se obvia la defensa de lo público. A tal respecto se señala la pertinencia de implantar nuevas fórmulas de control de calidad y nuevos estándares de trabajo - por ejemplo, trabajo por objetivos- entre otros. Todo esto desde la defensa de lo público.

"Para funcionar con un mínimo de garantías necesitamos que los servicios sociales sean fuertes. Habrá que trabajar más por objetivos, habrá que evaluar mucho más las políticas que se apoyan... Pero eso no significa que haya que dejar de apoyar al sistema público y fomentar el tercer sector. Habrá que trabajar en el sistema público con otros criterios de eficiencia, pero nunca ir en detrimento de lo público.” (E.r.45)

\section{Conclusiones}

Los países europeos están envueltos en un proceso de convergencia supranacional que los determina, causando una reestructuración notoria en sus políticas, incluidas las sociales.

En general, el análisis revela un importante cambio en los sistemas de bienestar que condiciona, e incluso determina, la intervención social. El creciente carácter restrictivo es uno de los aspectos más citados: un sistema marcado por las restricciones, los límites, el control, la falta de flexibilidad y un enfoque excesivamente burocrático. Este sistema restrictivo deja múltiples escenarios, situaciones y necesidades sin cobertura.

En relación con lo anterior, encontramos una línea discursiva mayoritaria que muestra su desacuerdo con respecto a estas medidas. No obstante, hallamos también una línea discursiva minoritaria que defiende la pertinencia del cambio, argumentado a través de la necesidad de reducir la dependencia ciudadana del modelo de bienestar.

En un contexto de gasto social limitado, la privatización y concertación de servicios sociales públicos se convierte en una nueva estrategia de reducción de costes. El rechazo a este tipo de medidas es prácticamente generalizado entre los/as expertos/as entrevistados/as, con argumentos tales como la pérdida de la calidad, la emergencia de los principios mercantiles en cuestiones sociales, o la desigualdad, segregación y polarización asociadas, entre otros. En resumen, la defensa de la responsabilidad pública constituye una máxima en los tres contextos y entre los diferentes grupos de interés.

Por último cabe destacar que, a lo largo del presente artículo, queda reflejado que los cambios en materia de bienestar social y servicios sociales no solo impactan en sus beneficiarios de estos sistemas, sino también en los gestores y prestadores. En esta línea, el análisis apunta que, en la actualidad, las disciplinas sociales se enfrentan a un sistema crecientemente restrictivo y limitador. Se hallan, por ende, marcadas por los recortes sociales, presupuestarios e institucionales, por el control, y por una orientación general crecientemente economicista y deshumanizadora. En definitiva, se ven obligas a redefinirse en la identificada como la "era de bronce" del bienestar social. 


\section{Bibliografía referenciada}

ATTAC-ESPAÑA (2004): Privatización de los servicios públicos en España, Madrid, Edición ATTAC.

FUNDACIÓN FOESSA (2014): VII Informe sobre exclusión social y desarrollo social en Andalucía y España, Madrid, Foessa.

LAS HERAS, P. (2002): "El Sistema Público de Servicios Sociales: contribución del Trabajo Social, desafíos, oportunidades y estrategias", Trabajo Social Hoy, 7-80.

GARCÍA-DOMINGO, M. Y SOTOMAYOR, E. (2016): "Escenarios desiguales de una Europa en crisis: aproximación a los efectos de la crisis socioeconómica en los Estados de Bienestar europeos: el caso de Alemania, Suecia y España”, Acta Sociológica; 69, pp. 181-205.

MISHRA, R. (1992): El Estado de bienestar en crisis: pensamiento y cambio social, Madrid, Ministerio de Trabajo y Seguridad Social.
MISHRA, R. (1996): “The welfare of nations" en BOYER R. y DRACHE D. (eds.): States against Markets. The Limits of Globalization, Londres, Routledge, pp. 316-333.

MORENO, L. (2001): Pobreza y exclusión. La “Malla de seguridad" en España, Madrid, CSIC Politeya.

NAVARRO, V. (1997): Neoliberalismo y Estado del bienestar, Barcelona, Ariel.

RODRÍGUEZ-CABRERO, G. (1989): “Política social en España: realidades y tendencias" en MUÑOZ R. (ed.), Crisis y futuro del Estado del Bienestar, Madrid, Alianza Editorial, pp. 183-204.

SUBIRATS, J. (2004): Pobreza y exclusión social. Un análisis de la realidad española y europea, Barcelona, La Caixa. 